\title{
Vacuum Pump System Diagnosis with the Pumping Speed Indicator
}

\author{
K. Lee \\ Department of Mechanical and Aerospace Engineering, Seoul \\ National University \\ Gwanak-ro 1, Gwanak-gu, Seoul, 151-742, Republic of Korea
}

\author{
G.H. Kim \\ Korea Atomic Energy Research Institute \\ 989-111 Daedeok-daero, Yuseong-gu, Daejeon 305-353, \\ Republic of Korea
}

\begin{abstract}
The diagnosis of a vacuum pump system for the semiconductor manufacturing is directly related to the failure rate of wafer. Pumping speed is the volume flow rate per unit time by the vacuum pump and represents the performance of vacuum pump directly. Unfortunately, it is impossible to measure the pumping speed in situation. We proposed an alternative method to infer pumping speed indirectly in situ. The Pumping speed indicator (PSI) is based on similarity of the pump-down method and the semiconductor manufacturing processes, estimates the exhaust capacity of vacuum pump.
\end{abstract}

Keywords-vacuum pump system; system diagnosis; pumping speed indicator

\section{INTRODUCTION}

The semiconductors should be manufactured in a clean condition. To remove the unnecessary chemical reactants and impurities, a number of vacuum pump system are used. So, the vacuum pump's performance directly affects the products failure rate. However, it is difficult to predict a suitable replacement point of vacuum pumps due to severe operating conditions and nonlinear characteristics. To avoid the production of off-spec products, developing the vacuum pump performance monitoring model is an important task from an economic point of view.

The performance of vacuum pump means the exhaust capacity of the pump. Pumping speed is the value indicating that directly. The measuring methods of the pumping speed of vacuum pumps are recommended as [1 3]. In order to measure the pumping speed accurately, the pump must be stopped and removed from the system and stabilized during a few hours at least. To overcome these disadvantages, advanced measuring method was proposed by [4]. But this method is also not fit for the purpose that measuring the real-time performance of the pump in the manufacturing processes. Therefore, the indicator to show the pumping speed without interrupting the production process is needed.

The semiconductor manufacturing process usually consists of two primary states and two transition sections (e.g., gas-loaded state; negative transition region; idle state, and positive transition region). The inlet pressure signals of negative transition region has exponential decay characteristic, and which is similar to the pump-down method: one of the pumping speed measurement methods. It is already identified that these exponential decay characteristic is directly relevant to the pumping speed. [5,6] the pump performance measuring methods using exponential decaying characteristics has been proposed in $[7,8]$

In this study, each exponential decaying characteristic is suggested as an indicator of the pumping speed. This pumping speed indicator method is applied to the data obtained in the field and verified that is suitable for the vacuum pump status diagnosis

\section{THEORETICAL BACKGROUND}

\section{A. Measurement of Pumping Speed}

The pumping speed is volume of gas which flows from the dome through the pump inlet per unit time, also called the volume flow rate. There are three primary methods for measuring the pumping speed (e.g., the throughput method, the orifice method and the pump-down method). Among them, the pump-down method is recommended when 4 variables, which are the volume of the test dome and two pressures before and after a pumping interval, are known.

For the measurement of the pumping speed with the pump-down method, all components of measuring devices are mounted together in accordance with Figure 1(A). The vacuum pump is connected to the test dome via the quick-acting valve and the pressure shell be measured with an absolute pressure vacuum gauge.

Figure 1(B) shows a pressure versus time curve for the pumping speed measurement. At the beginning, the initial pressure, $P_{t 1}$, drops quickly to $P_{t 1 w}$, by opening the quick-acting valve. The pressure is decreased from $P_{t 1 w}$ to $P_{t 2 w}$ by pumping during the fixed time interval, $\Delta t_{1}$. After closing the quick-acting valve, the pressure is stabilized to the value $P_{t 2}$ during the time interval, $\Delta t_{2}$, by thermal equalization. Then, pumping speed, $Q_{v}$, is calculated as Eq.1.

$$
Q_{v}=\frac{V+V_{i}}{\Delta t_{1}} \ln \frac{P_{t l w}}{P_{t 2 w}}
$$

To obtain accurate pumping speed with pump-down method, the pressure values are measured at the equilibrium state only. Then, $P_{t 1}$ and $P_{t 2}$ are actually measured pressure data; $P_{t 1 w}$ and $P_{t 2 w}$ are estimated values. The pressure of gas between the pump and the quick-acting valve is less than the pressure of the gas in the test dome. This causes a rapid expansion of gas in the dome when valve opens. So, the 
pressure of dome immediately after open the valve, $P_{t 1 w}$, can be calculated as Eq. 2.

$$
P_{t \mid w}=\frac{P_{t 1} V+P_{b 1} V_{i}}{V+V_{i}}
$$

where, $V$ is the volume of the test dome, $V_{i}$ is the volume between the pump and the quick-acting, $P_{b}$ is the base pressure of the pump.

If there is no leakage and gas desorption of the dome, the difference between $P_{t 2}$ and $P_{t 2 w}$ can be ignored. So Eq.1 can be corrected as Eq. 3.

$$
Q_{v}=\frac{V+V_{i}}{\Delta t_{1}} \ln \frac{P_{t t} V+P_{b 1} V_{i}}{\left(V+V_{i}\right) P_{t 2}}
$$
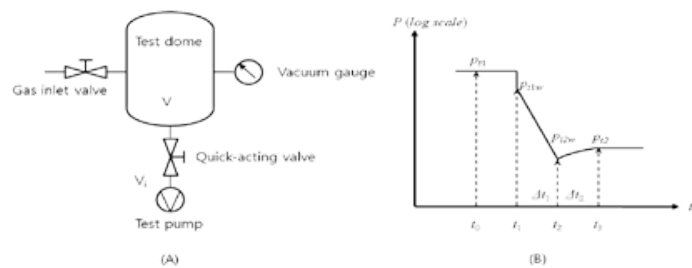

FIGURE I. THE PUMPING SPEED MEASUREMENT WITH PUMP-DOWN METHOD. (A) ARRANGEMENT FOR MEASURING. (B) PRESSURE VERSUS TIME CURVE OF PUMPING CYCLE.
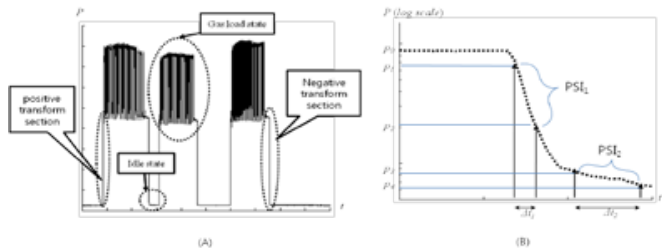

FIGURE II. Proposed the pumping speed indicator. (A) Inlet pressure data of Vacuum process, (B) Inlet pressure of Negative transform section.

\section{PUMPING SPEED INDICATOR}

As mentioned earlier, the pumping speed is utilized to diagnose the pump by monitoring of changes in the pumping speed. However, there are two fatal demerits. First, measuring pumping speed in situ is impossible because the pump should be disconnected from the manufacturing pump system to connect test dome. Second, measuring the pumping speed in real-time is impossible because it needs above a certain time for state equilibrium. It does not meet the diagnostic purpose finding out the appropriate replacement time without the interrupt of manufacturing progress. Therefore, we proposed the indirect indicators to diagnose the pumps system even if it does not represented the performance of the pump directly.

Figure 2(A) is the typical inlet pressure data of the vacuum pump during the semi-conductor manufacturing process. At the semiconductor manufacturing process, one pumping cycle usually consists of two primary states, the gas-loaded state and the idle state. While in the gas-loaded state, the vacuum pump aspirates $\mathrm{N}_{2}$ purge gas from the dome. During the idle state, the purge gas valve is closed and pump system works sustained. Between these two primary states, there are two transform sections; the positive-transform section and the negative-transform section. The transform section from the gas-loaded state to the idle state is named the negative-transform section. The physical property of this negative-transform section is quite similar to the pump-down method in that it can measure the pressure values during the gases of the dome taking out. In addition, as shown in Figure 2(B), there is a tendency to exponentially decrease during the negative transform section. Therefore, it is possible to diagnose the performance of the pump through comparison of the decreasing factors that appear same in every negative transform section.

As mentioned, variables used to determine the pumping speed, $Q_{v}$, are pressure, volume, and the time. First, pressure can be measured in real time from a pressure sensor attached to the inlet. We select CMR362 model of Pfeiffer Vacuum as pressure sensor and setup the sampling time as $0.1 \mathrm{~s}$. We cannot measure exact value of volume, $V$ and $V_{i}$. However, we can consider these values as constant, as it can be ignored that the volume change caused by temperature change during the manufacturing process. Then, it is possible to propose the pumping speed indicator (PSI), $Q_{v} /\left(V+V_{i}\right)$, instead of pumping speed $Q_{v}$. If PSI values of each manufacturing process are observed, present pump performance can be diagnosed by compared with present PSI and initial PSI.

At the normal operating process, the pressure of negative-transform section is shown as Figure 2(B). Generally, two apparent exponential decaying periods are observed. First period is $\Delta t_{1}$ during pressure dropped from $P_{1}$ to $P_{2}$ and second period is $\Delta t_{2}$ from $P_{3}$ to $P_{4}$, respectively. Pumping speed indicator from these two periods are $\mathrm{PSI}_{1}$ and $\mathrm{PSI}_{2}$. Generally, $P_{1}$ is about $90 \%$ of initial pressure $P_{0}$, and $P_{2}$ is about $30 \%, P_{3}$ is about $7 \%, P_{4}$ is about $1 \%$.[7,8]. However, we setup the first period from $80 \%$ to $40 \%$ of $P_{0}$ and second period from $5 \%$ to $2 \%$ of $P_{0}$ respectively to determine the margin for measurement. Therefore, PSI can be obtained as shown in Eq. 4.

$$
P S I_{1}=\frac{Q_{v 1}}{V+V_{i}}=\frac{1}{\Delta t_{1}} \ln \frac{P_{1}}{P_{2}}, \quad P S I_{2}=\frac{Q_{v 2}}{V+V_{i}}=\frac{1}{\Delta t_{2}} \ln \frac{P_{3}}{P_{4}}
$$

\section{Application of Field Data AND Verification}

\section{A. Application and Issues}

To verify whether the previously proposed pumping speed indicator can diagnose the state of the pump, PSI applied on the data obtained in the field. Using the measured data from the pump replaced due to degradation, PSI values are calculated during the manufacturing process. However, there is a problem to utilize the proposed PSI because of the abnormal negative-transform section. Figure 3(A) shows inlet pressure sample data measured for one day and every negative-transform sections are represented by $\nabla$ symbol. In this case, mainly two types of abnormal negative-transform observed. Abnormal type $\mathrm{A}$ is shown in the $3^{\text {rd }}$ and $6^{\text {th }}$ negative transform period of Figure 3 (A). As represented at Figure 3(C), pressure is momentarily increased without decreasing continuously. Therefore, abnormal type A means the leakage 
occurred case. Abnormal type B is shown in the $4^{\text {th }}, 8^{\text {th }}, 12^{\text {th }}$ negative transform period of Figure 3(A). As represented at Figure 3(D), proper pump-down doesn't happen, and normal gas-loaded state aren't processed because of problems in the process such as process cancel. Therefore, abnormal type B can be excluded from the record because actual manufacturing process did not occur. However, abnormal cases of type A cannot be excluded as manufacturing process is clearly made. The reason of leak is not clear whether due to intended leakage for manufacturing necessary or due to unintended malfunction of valve. Even if the reason of leak is a malfunction of valve, it doesn't mean that pump performance has problems. The purpose of this study is to propose a diagnostic method and verify techniques, we proposed the leak-determined PSI which can be deriving the performance of vacuum pump through the reselect of the measurement interval.

\section{B. Reset the PSI Obtained Section}

If the problem of type A is only a matter of valve and pump works perfectly, the pump down is made up entirely until leak expected point as represented at Figure 3(C). Therefore, if $d p / d t$ has a positive value during $\mathrm{PSI}_{1}$ interval, it is determined as an abnormal type A. then, the period of $\mathrm{PSI}_{1}$ set from $80 \%$ of $P_{0}$ point to the first occurrence of leak estimated point.

Figure 4 shows the results of $\mathrm{PSI}_{1}$ and $\mathrm{PSI}_{2}$ according to excluded type $\mathrm{B}$ and leak-determined type A. $\mathrm{PSI}_{1}$ is maintaining a constant level value during initial (phase 1), and is gradually decreasing according to manufacturing process repeated (phase 2). Especially, PSI1 indicate half value just before replacement (phase 3 ) compared to initial $\mathrm{PSI}_{1}$. In addition, $\mathrm{PSI}_{2}$ of the normal cases also decrease after certain period time (phase 1and 2). $\mathrm{PSI}_{2}$ sensed leak occurrence through about 2 time higher $\mathrm{PSI}_{2}$ value than $\mathrm{PSI}_{2}$ of normal case.

For the validation of the diagnostic model using PSI, we applied two more pump data set replaced due to exhaust performance problem. While Figure 4 represents pump case 1, Figure 5(A) and Figure 5(B) show the results of Pump case 2 and 3. Similar to the result of case 1(Figure 4), PSI kept fixed values while certain period time and pump are replaced after PSI decrease below a certain criteria. If manufacturing line is different, the model of pump and volume value $\left(V\right.$ and $\left.V_{i}\right)$ can be different. However, if the same type of pump used in the same line, the initial value of PSI has the same level value. So, if you know the PSI value of initial normal operating condition, you can make appropriate judgments pump replacement by performance degradation took place, when PSI value fall below the lower confidential limit (LCL). For example, the result of initial $\mathrm{PSI}_{1}$ and $\mathrm{PSI}_{1}$ of immediately preceding replacement from Figure 4 and 5 are given in Table 1.
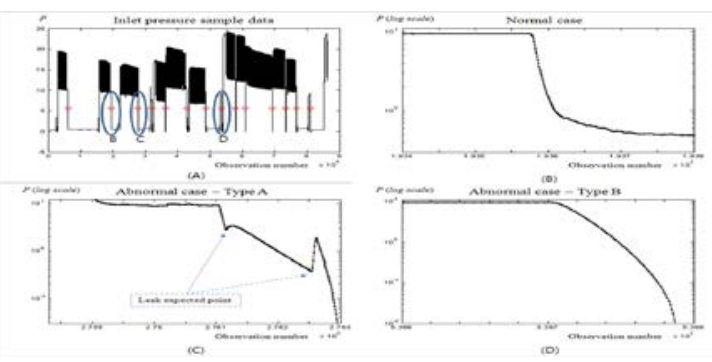

FIGURE III. (A) INLET PRESSURE SAMPLE DATA: 14 NEGATIVE TRANSFORM POINTS $(\nabla)$ ARE OBSERVED. THERE ARE NORMAL NEGATIVE TRANSFORM CASES AND TWO TYPES OF ABNORMAL NEGATIVE TRANSFORM CASES. (B) INLET PRESSURE OF NORMAL PUMP DOWN CASE: SECOND NEGATIVE TRANSFORM POINT OF (A). (C) INLET PRESSURE OF ABNORMAL PUMP DOWN CASE - TYPE A : THIRD NEGATIVE TRANSFORM POINT OF (A) AND OBSERVED THE OCCURRENCE OF GAS LEAK. (D) INLET PRESSURE OF ABNORMAL PUMP DOWN CASE - TYPE B: 8TH NEGATIVE TRANSFORM POINT OF (A) : THE GAS LOADED PROCESS DIDN'T COME INTO EXISTENCE. THIS TYPE B ABNORMAL PUMP DOWN OCCUR AT 4TH, 8TH, 12TH NEGATIVE TRANSFORM SECTION OF (A).

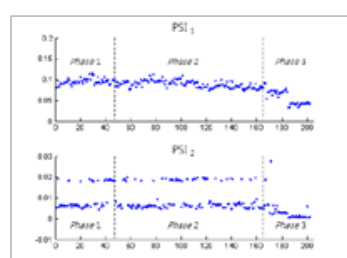

FIGURE IV. CHART OF PSI1 AND PSI2.

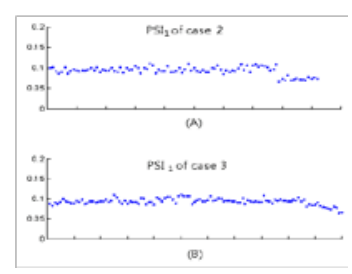

FIGURE IV. CHART OF PSI1 FROM CASE 2 AND CASE 3.
TABLE I .PSI OF PHASE 1 AND PHASE 3.

\begin{tabular}{cccc}
\hline \hline Case & $\begin{array}{c}\text { 1) } \text { PSI }_{1} \text { of } \\
\text { phase 1 }\end{array}$ & $\begin{array}{c}\text { (2) } \text { PSI }_{1} \text { of } \\
\text { phase 3 }\end{array}$ & (2) / (1) (\% ) \\
\hline Case 1 (4) & 0.0950 & 0.0458 & $48.2 \%$ \\
Case 2 (5-A) & 0.0975 & 0.0645 & $68.2 \%$ \\
Case 3 (5-B) & 0.0987 & 0.0657 & $66.6 \%$ \\
\hline \hline
\end{tabular}

\section{CONCLUSION}

In this study, pumipng speed indicator (PSI) is proposed instead of pumping speed Qv to diagnose the vacuum pump system during manufaturing process in situ. The performence diagnosis model using PSI was applied to vacuum pump data set. First, it is confirmed that PSI kept a fixed level of value. Second, when the pump system aged an digraded by repeated process, it is observed that PSI decreases near the replacement point. By monitoring PSI, vacuum pump performance can be seen how much decreased and when decreastion started. Through these things, it is assured that proposed pump system diagnistic model using PSI is meanigful. And user can determine a reasonable pump replacement point with PSI.

\section{REFERENCES}

[1] M. Hablanian, Recommended procedure for measuring pumping speeds, J. Vac. Sci. Technol. A, 5 (1987) 2552-2557 
[2] B. R. F. Kendall, Recommended procedures for measuring the performance of positive - displacement mechanical vacuum pumps, J. Vac. Sci. Technol. A, 7 (1989) 2403-2407

[3] ISO21360:2007(E), Vacuum Technology - Standard methods for measuring Vacuum-pump performance - General Description,(2007)

[4] W.S. Cheung, J.H. Shin, K.A. Park, and J.Y Lim, Precision vacuum pumping speed measurement using sonic nozzles, J. Vac. Sci. Technol. A 29(3), (2011)

[5] W Jitschin, K H Bernhardt, R Lachenmann, P Bickert and F J Eckle., Measuring the pumping speed of rough vacuum pumps: conventional stationary method vs intermittent pump-down method, 47, (1996) 505-509

[6] Nigel. S. Hariss, Modern vacuum pump practice, McGraw-Hill book company, London, 1989

[7] W.S. Cheung, J.Y. Lim, K.H. Chung, S. Lee, Trend monitoring and diagnostic analysis method and system for failure protection and for predictive maintenance of a vacuum pump, PCT/KR2004/003329

[8] W.S. Cheung, J.Y. Lim, K.H. Chung, S. Lee, Precision diagnostic method for the failure protection and predictive maintenance of a vacuum pump and a precision diagnostic system therefor, PCT/KR2004/003330 